\title{
Limits on light WIMPs with a germanium detector at 177 eVee threshold at the China Jinping Underground Laboratory
}

\author{
S.K. Liu, ${ }^{2,1}$ Q. Yue, ${ }^{1,}{ }^{7}$ K.J. Kang, ${ }^{1}$ J.P. Cheng, ${ }^{1}$ H.T. Wong,,${ }^{7}$ 'Y.J. Li, ${ }^{1}$ S.T. Lin, $,{ }^{2}, 7$ J.P. Chang, ${ }^{5}$ N. Chen, ${ }^{1}$ \\ Q.H. Chen, ${ }^{1}$ Y.H. Chen,${ }^{6}$ Y.C. Chuang, ${ }^{7}$ 円 Z. Deng, ${ }^{1}$ Q. Du, ${ }^{2}$ H. Gong, ${ }^{1}$ X.Q. Hao, ${ }^{1}$ H.J. He, ${ }^{1}$ Q.J. He, ${ }^{1}$ \\ H.X. Huang, ${ }^{3}$ T.R. Huang, ${ }^{7},{ }^{\dagger}$ H. Jiang, ${ }^{1}$ H.B. Li, ${ }^{7},{ }^{\dagger}$ J.M. Li,${ }^{1}$ J. Li, ${ }^{1}$ J. Li,${ }^{5}$ X. Li, ${ }^{3}$ X.Q. Li,${ }^{4}$ X.Y. Li,${ }^{4}$ Y.L. Li, ${ }^{1}$ \\ H.Y. Liao, ${ }^{7},{ }^{\dagger}$ F.K. Lin, ${ }^{7},{ }^{\dagger}$ L.C. Lü, ${ }^{1}$ H. Ma, ${ }^{1}$ S.J. Mao, ${ }^{5}$ J.Q. Qin, ${ }^{1}$ J. Ren, ${ }^{3}$ J. Ren, ${ }^{1}$ X.C. Ruan, ${ }^{3}$ M.B. Shen,${ }^{6}$ \\ L. Singh, ${ }^{7,8, \dagger}$ M.K. Singh, ${ }^{7,8, \dagger}$ A.K. Soma, ${ }^{7,8, \dagger}$ J. Su, ${ }^{1}$ C.J. Tang, ${ }^{2}$ C.H. Tseng, ${ }^{7,}$, J.M. Wang, ${ }^{6}$ L. Wang, ${ }^{1,2}$ \\ Q. Wang, ${ }^{1}$ S.Y. Wu ${ }^{6}$ Y.C. Wu,${ }^{1}$ Y.C. Wu,${ }^{5}$ Z.Z. Xianyu,${ }^{1}$ R.Q. Xiao, ${ }^{1}$ H.Y. Xing, ${ }^{2}{ }^{\text {F.Z. } X u},{ }^{1}$ Y. Xu,${ }^{4}$ X.J. Xu, ${ }^{1}$ \\ T. Xue, ${ }^{1}$ C.W. Yang, ${ }^{2}$ L.T. Yang, ${ }^{1}$ S.W. Yang,,${ }^{7}$ N. Yi,${ }^{1}$ C.X. Yu, ${ }^{4}$ H. Yu,${ }^{1}$ X.Z. Yu, ${ }^{2}$ X.H. Zeng, ${ }^{6}$ Z. Zeng, ${ }^{1}$ \\ L. Zhang, ${ }^{5}$ Y.H. Zhang, ${ }^{6}$ M.G. Zhao, ${ }^{4}$ W. Zhao, ${ }^{1}$ Z.Y. Zhou, ${ }^{3}$ J.J. Zhu, ${ }^{2}$ W.B. Zhu, ${ }^{5}$ X.Z. Zhu, ${ }^{1}$ and Z.H. Zhu ${ }^{6}$ \\ (CDEX Collaboration) \\ ${ }^{1}$ Key Laboratory of Particle and Radiation Imaging (Ministry of Education) \\ and Department of Engineering Physics, Tsinghua University, Beijing 100084 \\ ${ }^{2}$ College of Physical Science and Technology, Sichuan University, Chengdu 610064 \\ ${ }^{3}$ Department of Nuclear Physics, China Institute of Atomic Energy, Beijing 102413 \\ ${ }^{4}$ School of Physics, Nankai University, Tianjin 300071 \\ ${ }^{5}$ NUCTECH Company, Beijing 100084 \\ ${ }^{6}$ YaLong River Hydropower Development Company, Chengdu 610051 \\ ${ }^{7}$ Institute of Physics, Academia Sinica, Taipei 11529 \\ ${ }^{8}$ Department of Physics, Banaras Hindu University, Varanasi 221005
}

(Dated: August 18, 2018)

\begin{abstract}
The China Dark Matter Experiment reports results on light WIMP dark matter searches at the China Jinping Underground Laboratory with a germanium detector array with a total mass of $20 \mathrm{~g}$. The physics threshold achieved is 177 eVee ("ee" represents electron equivalent energy) at $50 \%$ signal efficiency. With $0.784 \mathrm{~kg}$-days of data, exclusion region on spin-independent coupling with the nucleon is derived, improving over our earlier bounds at WIMP mass less than $4.6 \mathrm{GeV}$.
\end{abstract}

PACS numbers: 95.35.+d, 98.70.Vc, 29.40.Wk

\section{INTRODUCTION}

Compelling evidence from astroparticle physics and cosmology indicates that dark matter constitutes about $27 \%$ of the energy density of our Universe 11. Weakly interacting massive particles (WIMPs, denoted by $\chi$ ) are the leading candidate for cold dark matter [2]. It is expected that WIMPs would interact with normal matter through elastic scattering. Direct detection of WIMPs has been attempted with different detector technologies [3. The anomalous excess of unidentified events at low energy with the DAMA 4, CoGeNT [5, CRESST-II 6] and CDMS ( $\mathrm{Si}$ ) [7] data has been interpreted as signatures of light WIMPs. They are however inconsistent with the null results from XENON 8, TEXONO [9], CDMSlite [10, LUX [11, SuperCDMS [12] and CDEX$1[13$ experiments. It is crucial to continue probing WIMPs with lower mass achievable by available techniques.

Our earlier measurements 13 have provided the first results on low-mass WIMPs from the China Dark Matter Experiment phase I (CDEX-1). With a $994 \mathrm{~g}$ pointcontact germanium detector, an energy threshold of 400

\footnotetext{
* Corresponding author: yueq@mail.tsinghua.edu.cn

$\dagger$ Participating as a member of TEXONO Collaboration
}

eVee was achieved. The experiment was performed at the China Jinping Underground Laboratory (CJPL) 14, which was inaugurated at the end of 2010 . With a rock overburden of more than $2400 \mathrm{~m}$ giving rise to a measured muon flux of $61.7 \mathrm{y}^{-1} \cdot \mathrm{m}^{-2}$ [15], CJPL provides an ideal location for low-background experiments.

We report final results of the "CDEX-0" experiment at CJPL, which is based on a pilot measurement with an existing prototype Ge detector with sub-keV energy threshold at a few gram modular mass. The experimental setup, candidate event selection procedures and constraints on WIMP-nucleon spin-independent elastic scattering are discussed in the subsequent sections.

\section{EXPERIMENTAL SETUP}

The CDEX-0 detector was previously used by the TEXONO experiment at a surface laboratory, where constraints on light WIMP were placed with a data set having a threshold of 220 eVee at 50\% signal efficiency [16]. The schematic design is depicted in Fig. 1. The NaI(Tl) crystal scintillator whose threshold is about $10 \mathrm{keVee}$ served as an anti-Compton (AC) detector which enclosed the cryostat. The thickness of its side is $48 \mathrm{~mm}$ and top is $130 \mathrm{~mm}$. The passive shielding system includes, from outside to inside, $1 \mathrm{~m}$ of polyethylene, $20 \mathrm{~cm}$ of lead, 20 


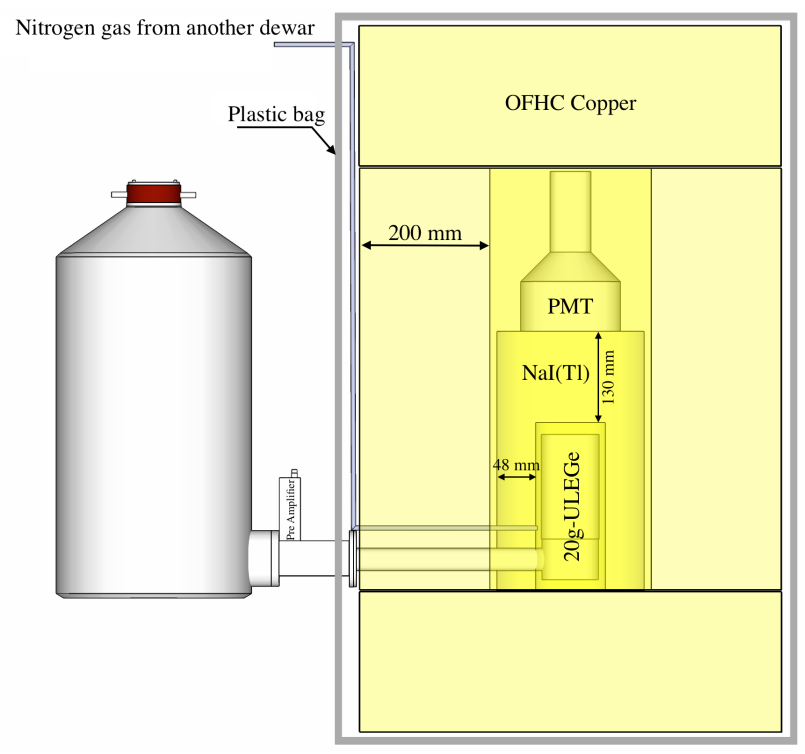

FIG. 1. Schematic diagram of the experimental setup which includes the germanium detector array and $\mathrm{NaI}(\mathrm{Tl})$ antiCompton detector, as well as the enclosing OFHC Cu shielding. The entire structure is placed inside a passive shielding system described in Ref. 14.

TABLE I. Measured parameters which characterize the resolution performance of the four crystals.

\begin{tabular}{ccccc}
\hline \hline $\begin{array}{c}\text { Crystal } \\
\text { number }\end{array}$ & $\begin{array}{c}\text { Pedestal } \\
\text { rms } \\
(\mathrm{eVee})\end{array}$ & $\begin{array}{c}\text { Noise } \\
\text { edge } \\
(\mathrm{eVee})\end{array}$ & $\begin{array}{c}\mathrm{FWHM}^{a} \\
\text { af }\end{array}$ 3.69 x rays & $\begin{array}{c}\text { Trigger efficiency } \\
\text { at } 50 \% \\
(\mathrm{eVee})\end{array}$ \\
\hline 1 & 51 & $\sim 300$ & $169 \pm 3$ & $171 \pm 5$ \\
2 & 33 & $\sim 180$ & $123 \pm 3$ & $100 \pm 2$ \\
3 & 64 & $\sim 400$ & $186 \pm 6$ & $267 \pm 3$ \\
4 & 68 & $\sim 250$ & $182 \pm 6$ & $146 \pm 4$ \\
\hline \hline
\end{tabular}

${ }^{\bar{a} \text { Full width at half maximum (FWHM). }}$

$\mathrm{cm}$ of borated polyethylene and $20 \mathrm{~cm}$ of OFHC (oxygenfree high-conductivity) copper. A plastic bag enclosing the OFHC copper was purged by nitrogen gas evaporated from the liquid nitrogen dewar. The detailed information about the passive shielding system was described in Ref. [14.

The $20 \mathrm{~g}$ ultra low energy threshold germanium (ULEGe) detector consists of four n-type crystals. Every crystal with an active mass of $5 \mathrm{~g}$ has a semiplanar configuration with a $\mathrm{p}^{+}$electrode on the outer surface, and $\mathrm{a} \mathrm{n}^{+}$contact of a small diameter is used as the central electrode, from which signals are derived. The surface electrode is of $\mu \mathrm{m}$ thickness fabricated by boron-ion implantation. The crystal array is encapsulated within the cryostat made of OFHC copper and the crystal center-tocenter spacing is $14 \mathrm{~mm}$. The cryostat end cap is made of carbon composite with the thickness of $0.6 \mathrm{~mm}$ allowing calibration with low-energy $\mathrm{x}$ rays outside.
The schematic diagram of the electronics and data acquisition (DAQ) system is shown in Fig. 2. The $\mathrm{n}^{+}$contact signal is read out by low noise FET in the vicinity of the Ge crystal and fed into a reset preamplifier. Each crystal has its respective preamplifier and two identical outputs distributed to two shaping amplifiers at 6 $\mu \mathrm{s}\left(\mathrm{SA}_{6}\right)$ and $12 \mu \mathrm{s}\left(\mathrm{SA}_{12}\right)$ shaping time. One output from $\mathrm{SA}_{6}$ was fed into the discriminator to supply the trigger for the DAQ system. The signals were sampled and recorded by a $100 \mathrm{MHz}$ flash analog-to-digital convertor (FADC). The recording time intervals were $70 \mu \mathrm{s}$ and $110 \mu$ s for the signals at $\mathrm{SA}_{6}$ and $\mathrm{SA}_{12}$, respectively. The photomultiplier tube (PMT) outputs from the AC detector at two different gain factors were also digitized. A veto period of $4 \mathrm{~ms}$ is applied after every preamplifier reset to reject electronic-induced noise [13]. Events provided by a random trigger (RT) with a pulse generator at $0.05 \mathrm{~Hz}$ were also recorded for calibration and DAQ dead time measurements. These RT events are also used to derive the efficiencies of those analysis selection procedures which are uncorrelated with the pulse shape of the Ge signals.

The relative timing of the $\mathrm{Ge}$ and $\mathrm{AC}$ detectors were recorded by standard time to digital convertors (TDC) with 25 ps resolution and a full range of $52 \mu \mathrm{s}$. In addition, the extended trigger time tag (ETTT) TDC extended the dynamic range to a full scale range up to 100 seconds maintaining the $25 \mathrm{ps}$ resolution. It is used to detect long-duration temporal correlations, such as those between the Ge and the reset signal.

At a total DAQ rate of $6.9 \mathrm{~Hz}$, the DAQ live time was measured to be $89.9 \%$ by RT events. The anomalously large dead time was due to inefficient methods of hardware synchronization in the prototype DAQ system, which were fixed in our subsequent data taking. The optimal area from $\mathrm{SA}_{6}$, which was defined as the light red shadow in Fig.6, was chosen as the energy measurements. Energy calibration was achieved by the external x-ray peaks from $\mathrm{Ca}, \mathrm{Mn}, \mathrm{Ti}, \mathrm{Cu}$ which were produced by the x-ray generator illuminating a mixture of these elements, as displayed in Fig. 3 [17.

The zero energy was defined by the random trigger events. The calibration uncertainties are $1.69 \mathrm{eVee}$ and 1.70 eVee at 130 eVee and 1 keVee, respectively. The measured parameters which characterize the resolution of the four crystals are shown in Table If. Crystal $\#_{2}$ provided the best performance and was adopted for subsequent analysis. The data taking interval spans over 174 days from November 24, 2012 to September 18, 2013 with interruptions due to laboratory construction and hardware failure, providing $0.784 \mathrm{~kg}$-days of physics data. Inclusion of data from the other crystals to the analysis would not provide substantial improvement to the final physics results.

Events in Ge crystal in coincidence (anticoincidence) with the $\mathrm{AC}$ detector are denoted as $\mathrm{AC}^{+(-)}$, respectively. Physics events induced by $\gamma$-rays are selected by the $\mathrm{AC}^{+}$tag and are used to optimize the selection cri- 


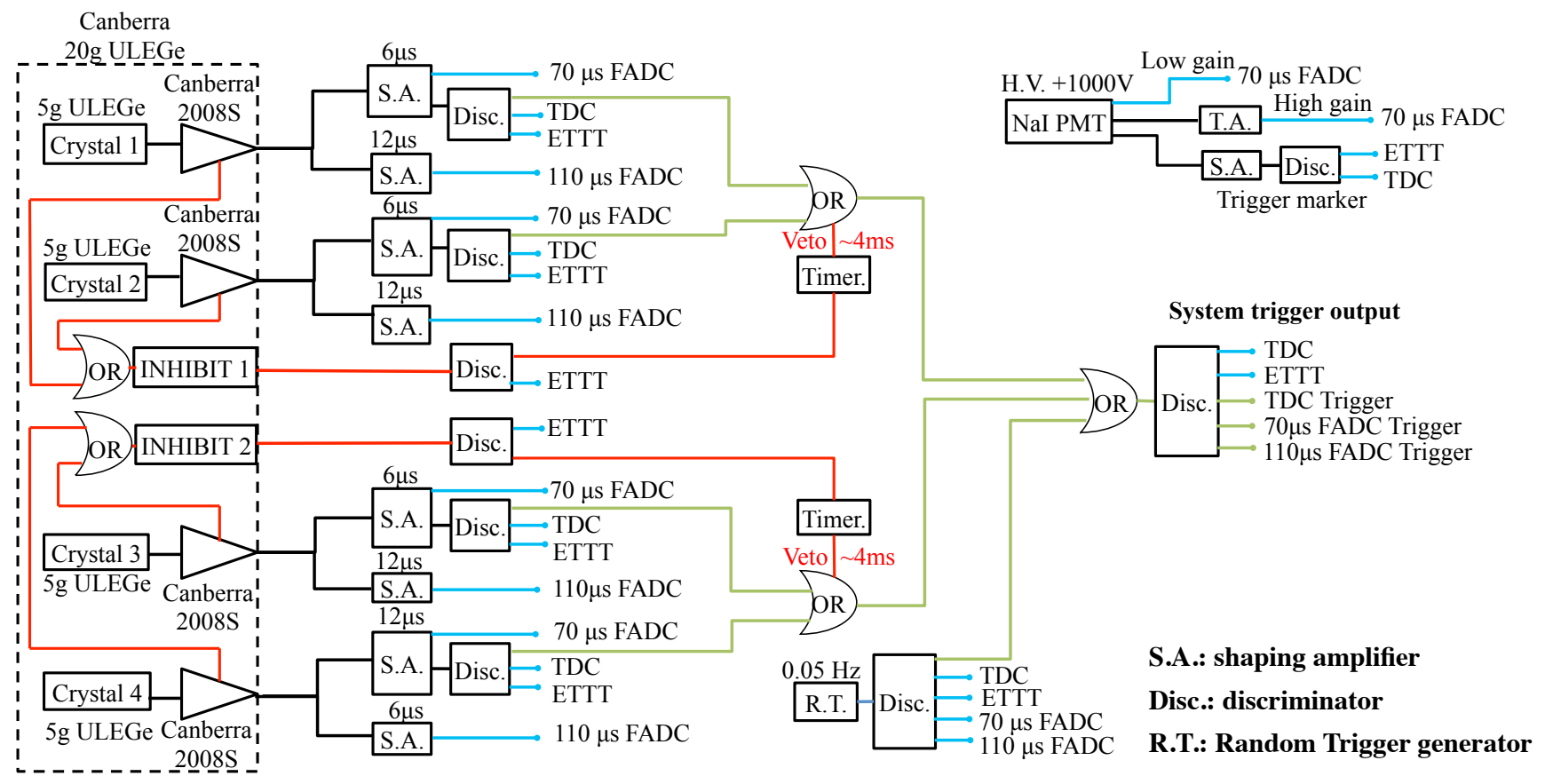

FIG. 2. Schematic diagram of the electronics and the DAQ system of the germanium array and the NaI(Tl) detector.

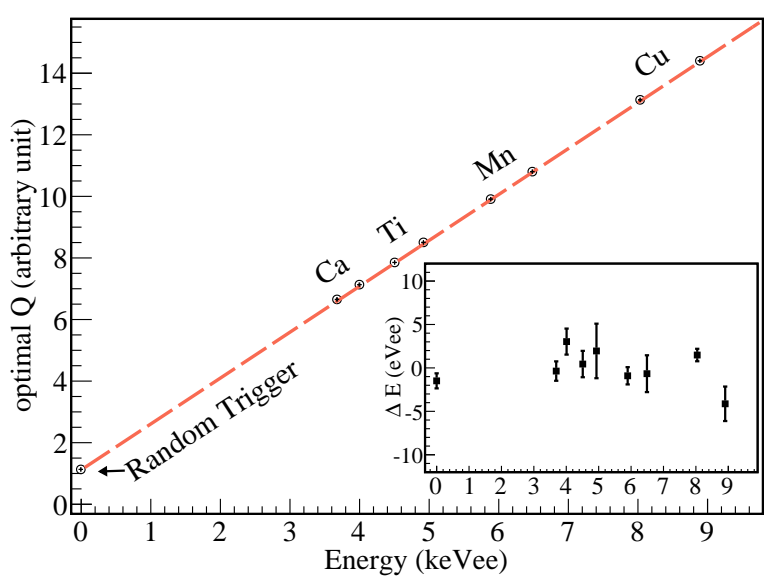

FIG. 3. Calibration line relating the optimal Q measurements from $\mathrm{SA}_{6}$ with the known energies from X-ray sources. The error bars are smaller than the data point size. The energy difference between the energy derived from the calibration and the real energy are depicted in the inset.

teria and to evaluate the signal efficiencies. Two complementary data sets were adopted. A ${ }^{60}$ Co source placed external to the NaI-AC detector provided high statistics data giving accurate measurement of the efficiencies, while the in situ low-background $\mathrm{AC}^{+}$data served as consistency cross-check.

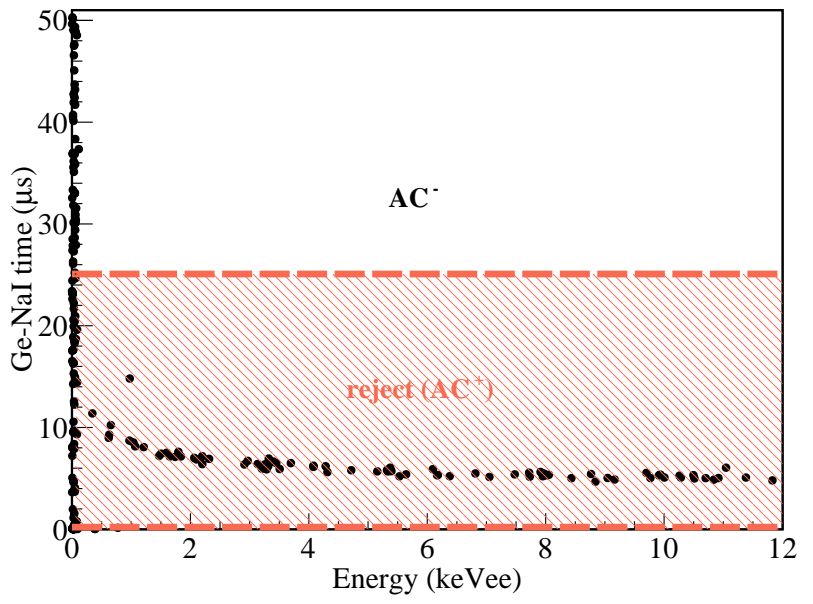

FIG. 4. Scatter plots of the difference between Ge and $\mathrm{NaI}(\mathrm{Tl})$ timing versus measured energy along with $\mathrm{AC}^{-}$selection and rejected parameter space.

\section{CANDIDATE EVENT SELECTION}

WIMP-induced interactions are characterized by being single-site events uncorrelated with other detector components, and having the same pulse shape as the events due to genuine physical processes. A series of data analysis criteria were adopted to select the $\chi \mathrm{N}$ events, and their corresponding signal efficiencies were measured. The details are discussed as follows, while the results are summarized in Table $\amalg$. 
(a)

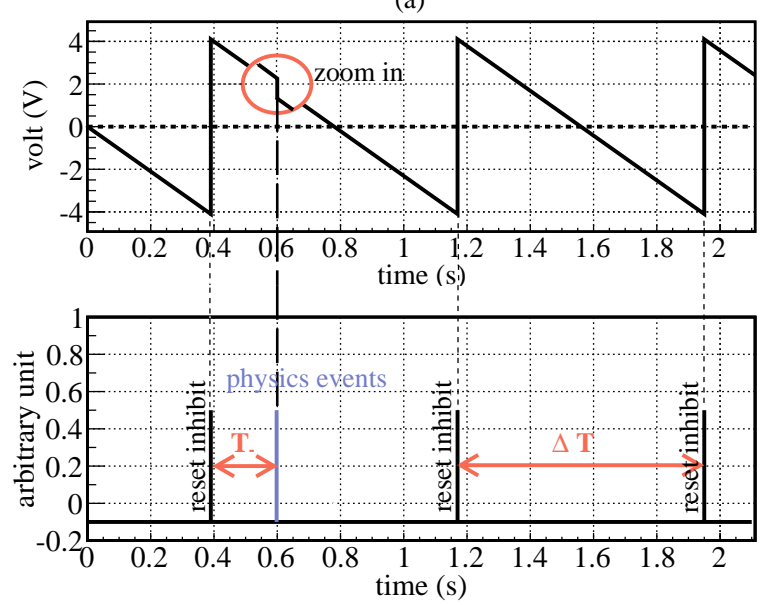

(b)

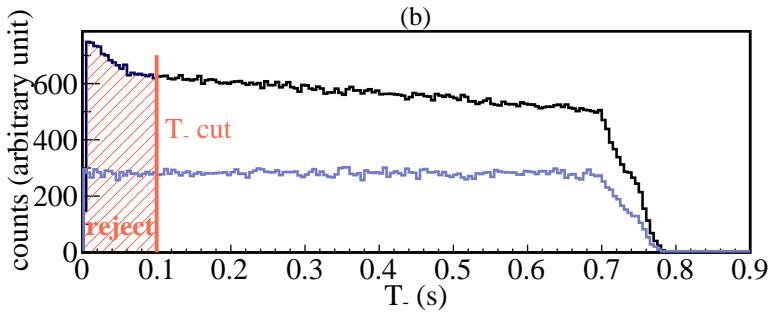

(c)

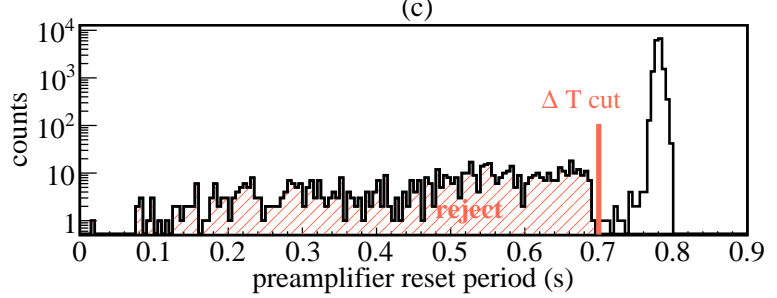

FIG. 5. (a) Raw signal from the reset preamplifier along with the timing of reset inhibit and a typical physics event. (b) The distributions of $\mathrm{T}_{\text {- for }}$ random trigger events (blue) and background events (black) are shown as well as the rejected parameter space. (c) The reset period cut and its rejected parameter space are displayed.

1. Timing (TM) selection: The preamplifier reset induces noise events with definite timing structure. The timing profiles of the reset and the events are illustrated in Fig. 5 (a). The timing distribution between an event and its previous reset, denoted by $\mathrm{T}_{-}$, is depicted in Fig. 5 (b) for physics events and RT. A cut of $\mathrm{T}_{-}<$ $0.1 \mathrm{~ms}$ removes all reset-induced background. The reset period $(\Delta \mathrm{T})$ distribution is shown in Fig. 5 (c). The duration in which at least five consecutive periods are persistently below $0.7 \mathrm{~s}$ is rejected. They correspond to temporary surge of leakage currents in the detector. The TM selection is applied to a subset of $38.6 \%$ of the data where the reset timing was recorded, in which the signal efficiency is derived from the survival probability of the RT events to be $77.1 \%$.

2. Anti-Compton $\left(\mathrm{AC}^{-}\right)$selection: The time difference between the AC and the Ge-trigger instant is depicted in

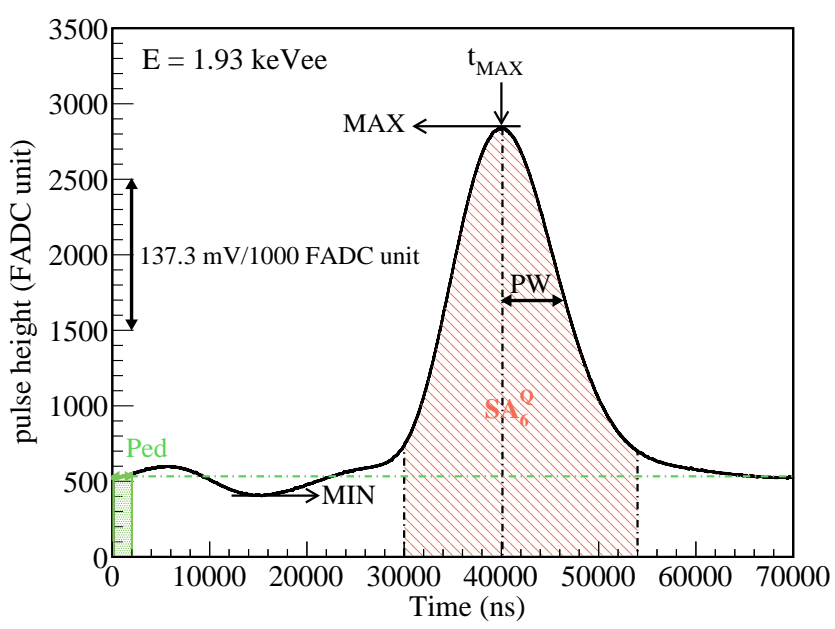

FIG. 6. Pulse shape parameters for PN Selection: Ped is the average of the first 200 time bins; MIN and MAX are the minima and maxima of the pulses, respectively, $t_{\text {MAX }}$ is the location of the maxima relative to the trigger instant and PW characterizes the pulse width. Energy is defined by the area $\mathrm{SA}_{6}^{\mathrm{Q}}$.

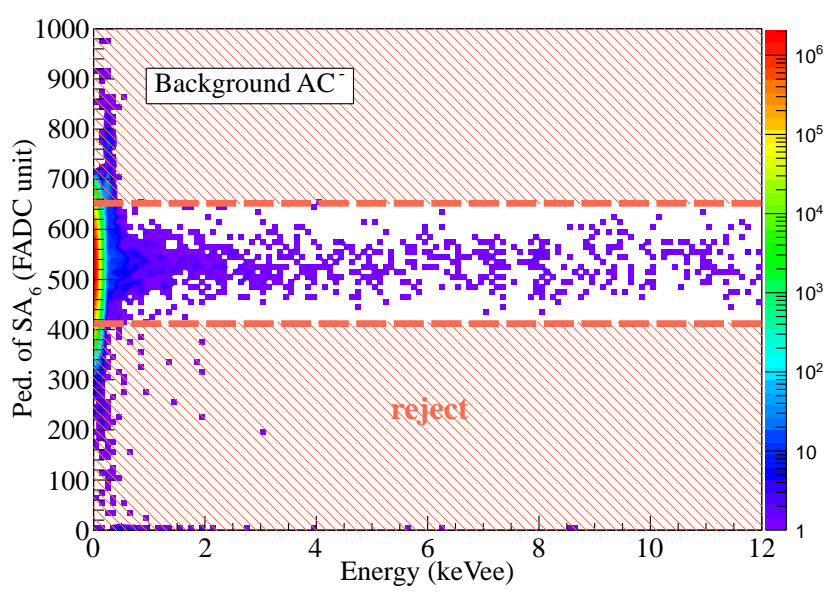

FIG. 7. Energy-independent $\mathrm{PN}_{\mathrm{i}}$ cut on Ped of $\mathrm{SA}_{6}$.

Fig. 4 The band corresponds to $\mathrm{AC}^{+}$events with coincidence of the Ge and NaI. The dependence with energy is due to the slow shaping pulse taking longer time at lower energy to cross a fixed threshold in the discriminator. The signal efficiency is $99.9 \%$ from the survival probability of the RT events.

3. Physics versus electronic noise (PN) selection: These events can be differentiated by their pulse shape parameters as defined in Fig. 6] where a 1.93 keVee particle pulse is shown. The energy-independent $\mathrm{PN}_{\mathrm{i}}$ selection is based on the pedestal (Ped) stability, as illustrated in Fig. 7. where the signal efficiencies are derived by the survival of the RT events to be $98.9 \%$. The energy-dependent $\mathrm{PN}_{\mathrm{d}}$ cuts make use of the minima (MIN) and maxima (MAX) of the pulse, the location of the maxima ( $t_{\text {MAX }}$ ) and the 

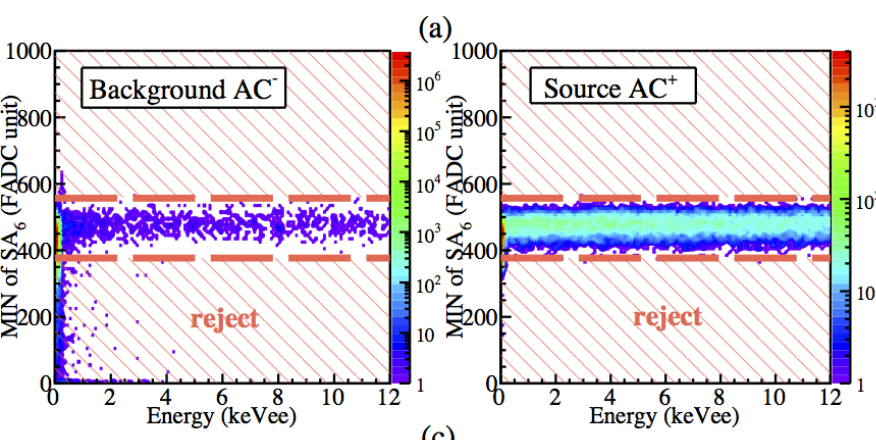

(c)
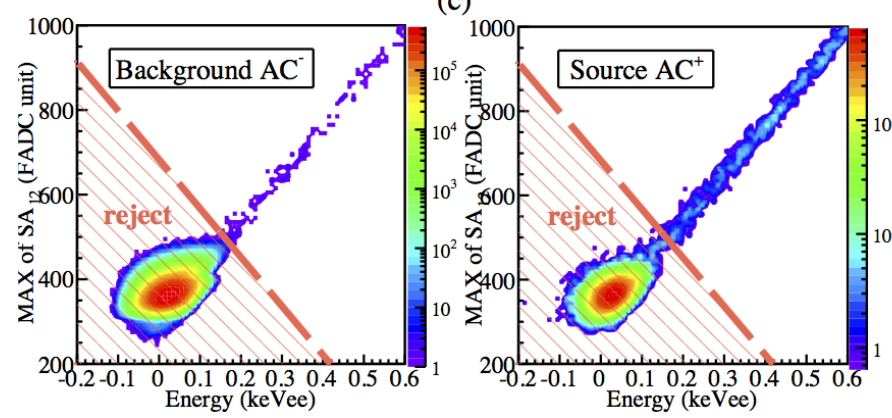
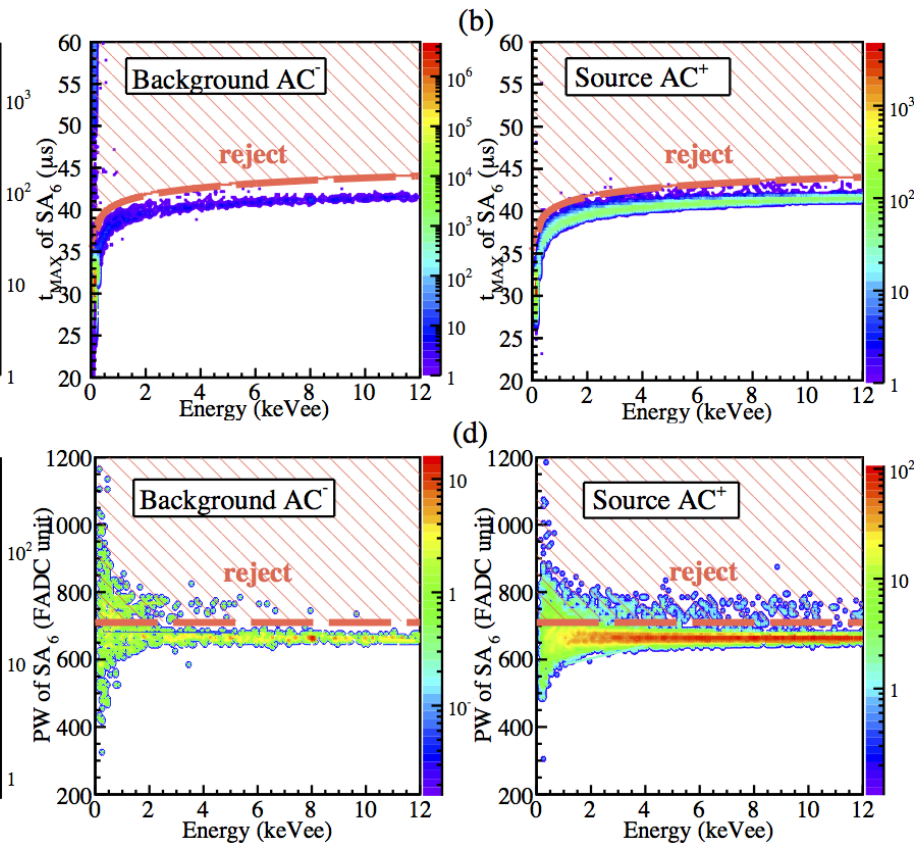

FIG. 8. The energy-dependent $\mathrm{PN}_{\mathrm{d}}$ cuts: (a) MIN cut, (b) t t MAX cut, (c) MAX cut and (d) PW cut, based on the parameters defined in Fig. 6

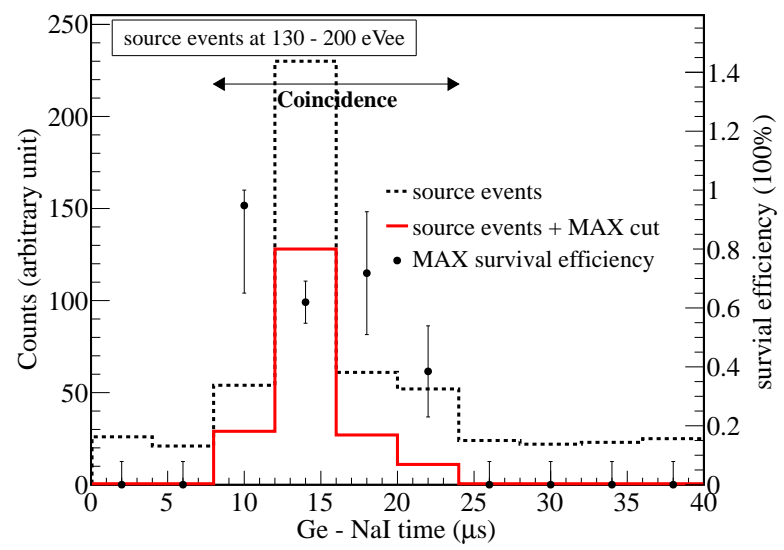

FIG. 9. The source events at 130-200 eVee versus the relative temporal distance between ULEGe triggers and AC signals are shown. The substantial value of survival efficiency at the coincidence time demonstrates the effectiveness of the MAX cut.

pulse width (PW), as depicted in Fig. 8 Events induced by physical processes have different distributions in these parameters from those of electronic noise. Physics events are defined by data taken with calibration ${ }^{60} \mathrm{Co} \gamma$ sources with $\mathrm{AC}^{+}$tag, also shown in Fig. 8. The selection parameter spaces are then applied to the candidate $\mathrm{AC}^{-}$ events. The survival probabilities of the $\mathrm{AC}^{+}$samples provide measurements of selection efficiencies.

While the MIN, $t_{\text {MAX }}$ and PW cuts would filter electronic noise and microphonics events, the MAX cut of
Fig. 8 (c) is the most important one to define the analysis threshold [16]. Physics and electronic noises events show different correlations between the area and amplitude of the pulse. The rejection criteria select physics events below the noise edge of 200 eVee, with efficiencies provided by the $\mathrm{AC}^{+}$samples. The timing distribution of $\mathrm{Ge}$ and $\mathrm{NaI}(\mathrm{Tl})$ for source events at 130-200 eVee energy is depicted in Fig. 9, before and after the MAX cut. Events outside the coincidence region are rejected at $100 \%$ efficiencies, showing the selection is indeed differentiating signal from electron noise events. Events in the coincidence range are kept, with the survival fractions representing the signal selection efficiencies. The variations with energy is depicted in Fig. 10 (a). Both hyperbolic tangent and error functions provide good and consistent descriptions to the data at the threshold region.

The trigger efficiencies are derived from the pulse shape of $\mathrm{SA}_{6}$. The amplitude and its rms are derived from the source $\mathrm{AC}^{+}$events at energy above the noise edge. The zero-energy point is defined by RT events, and interpolations are made for energy in between, assuming Gaussian distribution. The trigger efficiency is the fraction of the amplitude distribution above the discriminator threshold [16], as indicated in Fig. 10 (b). The 50\% trigger efficiency corresponds to $100 \pm 2$ eVee.

The combined efficiencies of all cuts (including trigger efficiency, TM, $\mathrm{PN}_{\mathrm{i}}, \mathrm{PN}_{\mathrm{d}}$ and $\mathrm{AC}^{-}$) are shown in Fig. 11. The physics threshold is $177 \pm 5$ eVee corresponding to a combined efficiency at $50 \%$. The signal efficiencies and background suppression factors at threshold and at a high-energy bin are summarized in Table II 
(a)

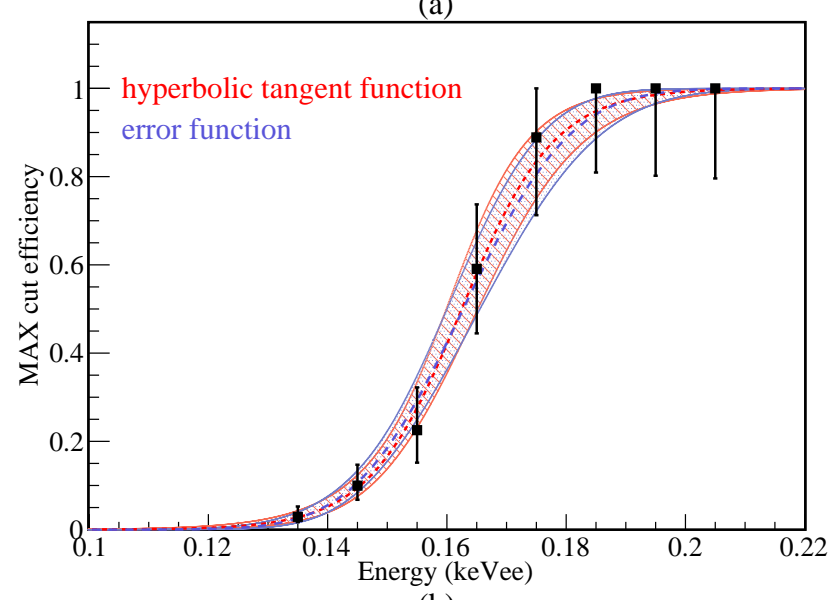

(b)

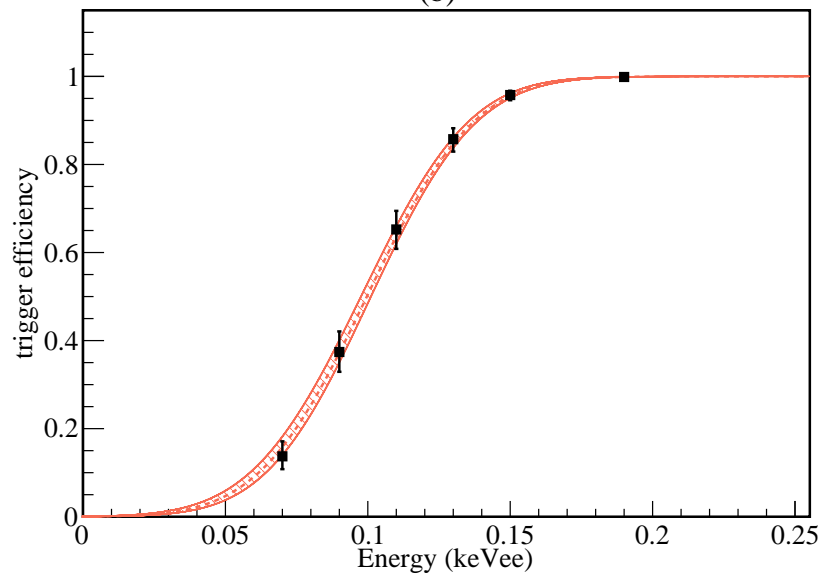

FIG. 10. (a) MAX cut efficiencies with two different fitting functions. (b) Trigger efficiencies derived from the source $\mathrm{AC}^{+}$events.

to illustrate and compare the effects of each process.

\section{CONSTRAINTS ON LIGHT WIMPS}

The measured raw spectra and those at different stages of the analysis are depicted in Fig. 12. Standard error propagation formulas are adopted, using the statistical uncertainties of the raw measurements as well as those on selection efficiencies from Fig. 11. The minimum energy is at 130 eVee, matching the first finite efficiency bin of Fig. 11. The only explicit structure observed was the $8.041 \mathrm{keV}$ copper $\mathrm{K} \alpha \mathrm{x}$ ray. They are produced from the interactions of high-energy $\gamma$ rays on the copper support structures in the vicinity of the Ge crystal, and hence a portion of these events are tagged by the $\mathrm{AC}^{-}$selection. The spectrum of events that survived all selection criteria is flat above $1.5 \mathrm{keVee}$, due to ambient radioactivity of high-energy $\gamma$ rays. The residual spectrum after subtraction of this background channel is depicted in the inset of Fig. 12 .

The energy spectra due to $\chi \mathrm{N}$ spin-independent in-

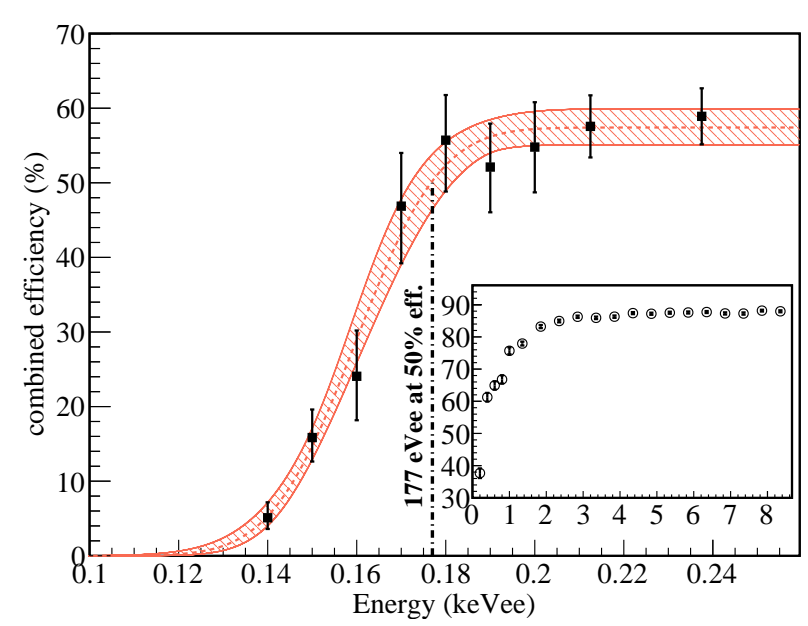

FIG. 11. The combined efficiencies in the low energy range and in an extended energy range are depicted respectively. In the latter one the error bars are smaller than the data point size.

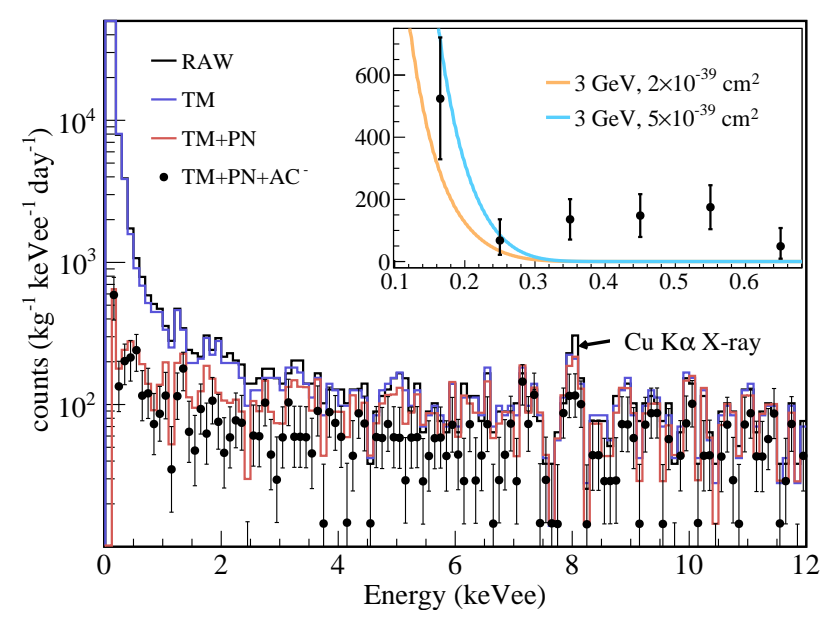

FIG. 12. Measured energy spectra of 20g-ULEGe, showing the raw spectra and those at the different stages of the analysis. The inset figure shows the low-energy spectrum after subtraction of a flat background due to high-energy $\gamma$ rays, superimposed with the predicted spectra for $3 \mathrm{GeV}$ WIMPs with $\sigma_{\chi \mathrm{N}}^{\mathrm{SI}}=2 \times 10^{-39} \mathrm{~cm}^{2}$ and $\sigma_{\chi \mathrm{N}}^{\mathrm{SI}}=5 \times 10^{-39} \mathrm{~cm}^{2}$.

teractions cannot be larger than the residual spectrum. The thickness of the surface inactive layer [18] is only tens of micrometers and can be neglected. Upper limits on their cross sections $\left(\sigma_{\chi \mathrm{N}}^{\mathrm{SI}}\right)$ as a function of WIMP mass are derived, using the binned Poisson method [19]. The input parameters include quenching factor provided by the TRIM program [20], coupled with a $10 \%$ systematic error implied by the spread of the measured data at the recoil energy of $254 \mathrm{eV}$ to $10 \mathrm{keV}$, standard WIMP halo assumption [21, conventional astrophysical models (local WIMP density of $0.3 \mathrm{GeV} \cdot \mathrm{cm}^{-3}$ and Maxwellian velocity distribution with $\nu_{0}=220 \mathrm{~km} \cdot \mathrm{s}^{-1}$, the escape 
TABLE II. Summary of candidate event selection procedures at two representative energy intervals. Listed are the individual and cumulative background survival fraction $[\lambda(\%)$ and $\Pi \lambda(\%)$, respectively] and the candidate signal efficiency $[\epsilon(\%)]$, as well as "Combined Efficiency" multiplying all efficiency factors together including trigger efficiency, TM, $\mathrm{PN}_{\mathrm{i}}$, $\mathrm{PN}_{\mathrm{d}}$ and $\mathrm{AC}^{-}$efficiencies.

\begin{tabular}{|c|c|c|}
\hline Energy bin & $130-200$ eVee & $600-700$ eVee \\
\hline Raw background counts & 5329 & 62 \\
\hline DAQ dead time $(\%)$ & \multicolumn{2}{|c|}{$10.1 \pm 0.2$} \\
\hline Trigger efficiency (\%) & $96.6 \pm_{1.1}^{0.9}$ & 100 \\
\hline \multicolumn{3}{|c|}{ Timing Selection } \\
\hline$\lambda[\Pi \lambda](\%)$ & $95.8[95.8]$ & $79.0[79.0]$ \\
\hline$\epsilon(\%)$ & \multicolumn{2}{|c|}{$77.1^{a} \pm 0.2$} \\
\hline \multicolumn{3}{|c|}{$\mathrm{PN}_{\mathrm{i}}$ cuts } \\
\hline$\lambda[\Pi \lambda](\%)$ & $92.5[88.6]$ & $73.5[58.1]$ \\
\hline$\epsilon(\%)$ & \multicolumn{2}{|c|}{$98.9 \pm 0.2$} \\
\hline \multicolumn{3}{|c|}{$\mathrm{PN}_{\mathrm{d}}$ cuts } \\
\hline \multicolumn{3}{|l|}{ MAX cut: } \\
\hline$\lambda[\Pi \lambda](\%)$ & $2.0[1.8]$ & $100[58.1]$ \\
\hline$\epsilon(\%)$ & $53.9 \pm \pm_{5.0}^{4.5}$ & $100 \pm 0.0$ \\
\hline \multicolumn{3}{|l|}{ MIN cut: } \\
\hline$\lambda[\Pi \lambda](\%)$ & $38.0[0.7]$ & $69.4[40.3]$ \\
\hline$\epsilon(\%)$ & $95.4 \pm 1.5$ & $99.8 \pm 0.2$ \\
\hline \multicolumn{3}{|l|}{$t_{\text {MAX }}$ cut: } \\
\hline$\lambda[\Pi \lambda](\%)$ & $27.0[0.5]$ & $50.0[29.0]$ \\
\hline$\epsilon(\%)$ & $96.3 \pm 1.4$ & $93.1 \pm 1.1$ \\
\hline \multicolumn{3}{|l|}{ PW cut: } \\
\hline$\lambda[\Pi \lambda](\%)$ & $40.7[0.2]$ & $55.6[16.1]$ \\
\hline$\epsilon(\%)$ & $71.7 \pm 3.4$ & $79.2 \pm 1.9$ \\
\hline \multicolumn{3}{|l|}{ Combined $\mathrm{PN}_{\mathrm{d}}$ cuts: } \\
\hline$\lambda[\Pi \lambda](\%)$ & $0.2[0.2]$ & $27.8[16.1]$ \\
\hline$\epsilon(\%)$ & $35.5 \pm_{3.8}^{3.5}$ & $73.6 \pm 2.0$ \\
\hline \multicolumn{3}{|c|}{ Anti-Compton Selection } \\
\hline$\lambda[\Pi \lambda](\%)$ & $90.9[0.2]$ & $60.0[9.7]$ \\
\hline$\epsilon(\%)$ & \multicolumn{2}{|c|}{$100.0 \pm 0.2$} \\
\hline Combined Efficiency $(\%)$ & $30.9 \pm_{3.3}^{3.0}$ & $66.3 \pm 1.8$ \\
\hline After-all-cuts counts & 10 & 6 \\
\hline $\begin{array}{l}\text { After-all-cuts rate } \\
\left(\mathrm{kg}^{-1} \mathrm{keV}^{-1} \mathrm{day}^{-1}\right)\end{array}$ & $590 \pm_{197}^{195}$ & $115 \pm_{39}^{58}$ \\
\hline
\end{tabular}

$\overline{\bar{a}}$ applied to a subset of $38.6 \%$ of the data.

velocity $\nu_{\mathrm{esc}}=544 \mathrm{~km} \cdot \mathrm{s}^{-1}$ ) and energy resolution of detector derived from the calibration data.

The exclusion curve at $90 \%$ confidence level is shown in Fig. 13, together with those of several selected experiments 413, 16, 22. The previous results of CRESSTI 22] and TEXONO [16 are reanalyzed using the currently-favored astrophysical parameters. Under this consistent analysis, this result improves over our earlier bounds from the same detector at a surface location 16 extending the low reach of light WIMPs to $2 \mathrm{GeV}$, and over the published limit [10] at $\mathrm{M}_{\chi}<3.5 \mathrm{GeV}$. The predicted $\chi \mathrm{N}$ recoil spectra due to the allowed (excluded) $\sigma_{\chi \mathrm{N}}^{\mathrm{SI}}$ at $\mathrm{m}_{\chi}=3 \mathrm{GeV}$ are superimposed with the residual spectrum in the inset of Fig. 12 .

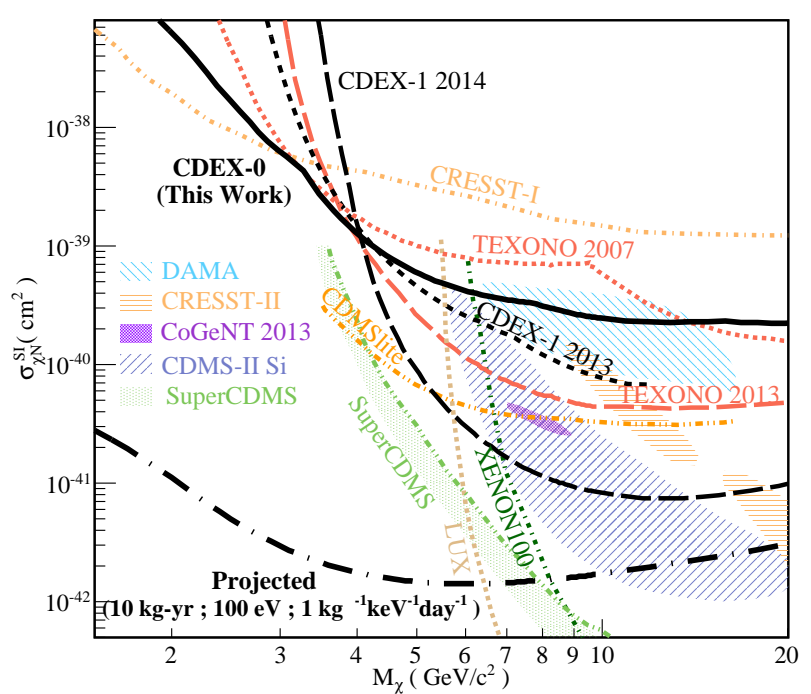

FIG. 13. Exclusion plot of spin-independent $\chi \mathrm{N}$ coupling at $90 \%$ confidence level, superimposed with the results from other benchmark experiments. Allowed regions given by CoGeNT [5], DAMA/LIBRA [4, CDMS-II (Si) 7] and CRESST-II [6] are presented, as well as the exclusion limits from XENON100 8, TEXONO 9, 16, CDMSlite [10, LUX [1], SuperCDMS [12], CDEX-1 [13] and CRESST-I 22]. The potential reach at indicated projected sensitivities with pointcontact germanium detectors is also displayed.

\section{SUMMARY AND PROSPECTS}

The results presented in this article correspond to the first completed program of the pilot experiment at the new underground facility CJPL. Improved constraints are derived with a conventional Ge detector of good threshold response but only a few gram modular target mass. Novel p-type point-contact germanium detectors were developed in the past few years 23 , offering sub$\mathrm{keV}$ energy threshold with kg-scale target such that the background level per unit mass is greatly reduced due to self-attenuation effects. Dark matter experiments with this detector technique are being pursued at CJPL 13 . and elsewhere [5, 9]. The projected sensitivities of the realistic benchmark sensitivities of 100 eVee threshold at $1 \mathrm{~kg}^{-1} \mathrm{keV}^{-1}$ day $^{-1}$ background level for $10 \mathrm{~kg}$-year exposure is overlaid in Fig. 13 . 


\section{ACKNOWLEDGMENTS}

This work was supported by the National Natural Science Foundation of China (Contracts No.10935005,
No. 10945002, No. 11275107 and No. 11175099) and National Basic Research program of China (973 Program) (Contract No. 2010CB833006) and NSC 99-2112-M-001017-MY3, and the Academia Sinica Principle Investigator Award 2011-2015 from Taiwan.
[1] J. Beringer et al., Phys. Rev. D 86, 010001 (2012). and references therein; P. A. R. Ade et al., (2013) arXiv:1303.5076.

[2] C. Kelso, D. Hooper, and M. R. Buckley, Phys. Rev. D 85, 043515 (2012), and references therein.

[3] J. D. Lewin and P. F. Smith, Astropart. Phys. 6, 87 (1996)

[4] P. Belli et al., Phys. Rev. D 84, 055014 (2011), R. Bernabei et al., Eur. Phys. J. C 67, 39 (2010).

[5] C. E. Aalseth et al., Phys. Rev. Lett. 106, 131301 (2011). Phys. Rev. D 88, 012002 (2013); arXiv:1401.3295v1

[6] G. Angloher et al., Eur. Phys. J. C 72, 1971 (2012).

[7] R. Agnese et al., Phys. Rev. Lett. 111, 251301 (2013)

[8] E. Aprile et al., Phys. Rev. Lett. 109, 181301 (2012).

[9] H. B. Li et al., Phys. Rev. Lett. 110, 261301 (2013) Astropart. Phys. 56, 1 (2014).

[10] R. Agnese et al., Phys. Rev. Lett. 112, 041302 (2014)

[11] D. S. Akerib et al., Phys. Rev. Lett. 112, 091303 (2014).

[12] R. Agnese et al., Phys. Rev. Lett. 112, 241302 (2014)

[13] W. Zhao et al., Phys. Rev. D 88, 052004 (2013) K. J. Kang et al., Chinese Phys. C 37, 126002 (2013); Q. Yue et al., (2014), arxiv:1404.4946.
[14] K. J. Kang et al., Front. Phys. 8, 412 (2013)

[15] Y. C. Wu et al., Chin. Phys. C 37, 086001 (2013)

[16] S. T. Lin et al., Phys. Rev. D 79, 061101 (2009).

[17] Y. C. Wu et al., J Tsinghua Univ (Sci \& Technol) 53, 1365 (2013).

[18] U. Tamm, W. Michaelis, and P. Coussieu, Nucl. Instrum. Methods 48, 301 (1967); M. G. Strauss and R. N. Larsen, 56, 80 (1967); E. Sakai, IEEE Trans. Nucl. Sci. 18, 208 (1971).

[19] C. Savage, G. Gelmini, P. Gondolo, and K. Freese, J. Cosmol. Astropart. Phys. 04, 010 (2009)

[20] J. F. Ziegler, Nucl. Instrum. Methods Phys. Res., Sect. B 219-220, 1027 (2004) S. T. Lin et al., |arXiv:0712.1645v4

[21] F. Donato, N. Fornengo, and S. Scopel, Astropart. Phys. 9, 247 (1998)

[22] G. Angloher et al., Astropart. Phys. 18, 43 (2002).

[23] P. N. Luke, F. S. Goulding, N. W. Madden, and R. H. Pehl, IEEE Trans. Nucl. Sci. 36, 926 (1989) P. S. Barbeau, J. I. Collar, and O. Tench, J. Cosmol. Astropart. Phys. 09, 009 (2007). 